\title{
LDPC-Coded OFDM for Optical Communication Systems with Direct Detection
}

\author{
Ivan B. Djordjevic, Member, IEEE, Bane Vasic, Senior Member, IEEE, and Mark A. Neifeld, Member, IEEE
}

\begin{abstract}
Low-density parity-check (LDPC) coded orthogonal frequency division multiplexing (OFDM) is proposed as an efficient coded modulation technique suitable for optical communication systems. We show that in long-haul fiber optics communications, LDPC-coded OFDM increases spectral efficiency, and reduces the influence of residual chromatic dispersion and the influence of fiber nonlinearities. LDPC-coded OFDM is as also a promising polarization-mode dispersion (PMD) compensation technique. In free space optical communications, LDPC-coded OFDM improves the immunity to the atmospheric turbulence; while in multimode fiber links, it reduces the influence of intermodal dispersion. In plastic optical fiber links it reduces the influence of dispersion and helps to overcome the bandwidth limitations. Several power efficient OFDM schemes and LDPC codes suitable for use in LDPCcoded OFDM optical communication systems are introduced.
\end{abstract}

Index Terms-Fiber optics communications, free space optical communications, graded-index plastic optical fiber links, lowdensity parity-check (LDPC) codes, optical communications, orthogonal frequency division multiplexing (OFDM).

\section{INTRODUCTION}

$\mathbf{O}$ RTHOGONAL frequency division multiplexing (OFDM) [1]-[8] is a special case of multicarrier transmission in which a single information-bearing stream is transmitted over many lower rate subchannels. OFDM has already been used in a variety of applications such as digital audio broadcasting [1], high-definition television (HDTV) broadcasting [2], high bitrate digital subscriber line (DSL), asymmetric DSL and very high-data rate DSL, IEEE 802.11, and multimedia mobile access communications wireless LANs [1]. It has recently been proposed for use in radio-over-fiber-based links [3], in freespace optical communications [4], in long-haul optical communications systems [5], [6], in multimode fiber links [7], and as a multiplexing method to enable 100-Gb/s Ethernet [8]. Due to the orthogonality among subcarriers in OFDM, partial overlap of neighboring frequency slots is allowed, thereby improving spectral efficiency as compared with a conventional multicarrier system. Also, by using a sufficiently large number of subcarriers and cyclic extension, the intersymbol interference (ISI) due to dispersion can be significantly reduced. Moreover, a simple equalization technique for chromatic dispersion compensation proposed in [5] and [7] can be easily adopted for use in bandwidth limited channels such as plastic optical fiber (POF) links.

Manuscript received December 15, 2006; revised May 24, 2007. This work was supported in part by the National Science Foundation (NSF) under Grant IHCS 0725405 and Grant ITR 0325979.

The authors are with the Department of Electrical and Computer Engineering, University of Arizona, Tucson, AZ 85721 USA. (e-mail: ivan@ece.arizona.edu; vasic@ece.arizona.edu; mark@ece.arizona.edu).

Digital Object Identifier 10.1109/JSTQE.2007.901893
In this paper, we show that low-density parity-check (LDPC) coded OFDM is an efficient coded modulation technique suitable for use in different optical communication systems: 1) graded-index POF (GI-POF) links to deal with dispersion and to overcome bandwidth limitations; 2) free-space optical (FSO) communications to improve immunity to atmospheric turbulence; 3) long-haul fiber-optics communications to increase the spectral efficiency, for chromatic dispersion and polarizationmode dispersion (PMD) compensation, and to reduce the influence of fiber nonlinearities by operating at lower symbol rate; 4) $100-\mathrm{Gb} / \mathrm{s}$ Ethernet; and 5) multimode fiber links to overcome intermodal dispersion.

The first type of the systems considered in this paper is the optical communication system over GI-POF link. GI-POF is a robust, low-cost, and easy to install transmission medium [9]-[11]. Possible applications of GI-POF include gigabit-Ethernet and uncompressed HDTV [11]. However, due to limited bandwidthlength product of GI-POF (about $3.45 \mathrm{GHz} \times 100 \mathrm{~m}$, see [9]), these fibers impose serious bandwidth limitations for applications at $2.5 \mathrm{~Gb} / \mathrm{s}$ and above. To counter the bandwidth problem an adaptive multiple subcarrier operating at $1 \mathrm{~Gb} / \mathrm{s}$ with spectral efficiency of $6.3 \mathrm{~b} / \mathrm{s} / \mathrm{Hz}$, and employing either 256-QAM or 64-QAM was proposed in [11]. Unfortunately, as shown in [1], the multiple subcarrier scheme is not spectrally efficient because it requires guard bands between the neighboring subbands. The multiple subcarrier systems are also not particularly good at dealing with ISI introduced by limited GI-POF bandwidth. Moreover, the multiple subcarrier scheme proposed in [11] is power-inefficient because it requires the use of a large DC bias to obtain a nonnegative signal [i.e., for transmission over an intensity modulation/direct detection (IM/DD) link]. As we will show the OFDM can improve spectral efficiency and ISI tolerance to chromatic dispersion of a multiple subcarrier (multicarrier) scheme [11]. In addition, to improve poor power efficiency of multiple subcarrier systems a single-sideband (SSB) transmission and clipped- or unclipped-OFDM is used. Due to severe dispersion experienced by signals at $10-\mathrm{Gb} / \mathrm{s}$ (and higher) transmitted over GI-POF link a powerful forward error correction (FEC) is required. Several such FEC schemes based on girth-8 low-density parity-check (LDPC) codes will be discussed herein.

The second system that can benefit from OFDM considered in this paper is a free-space optical communication system. Due to the high complexity associated with coherent detection, current FSO systems [13], [14] employ IM/DD. Such systems use pointto-point communication between the two optical transceivers along a line of sight. The IM/DD technique is also used in state-of-the-art fiber-optic communications, and the availability of optical components used in fiber-based systems makes FSO 
communication a cost-effective solution for high-rate image, voice, and data transmission [13]. However, an optical wave propagating through the air experiences fluctuations in amplitude and phase due to atmospheric turbulence [15]. The atmospheric turbulence is caused by variations in the refractive index of the transmission medium due to inhomogenities in temperature and pressure caused by solar heating and wind. In this paper, we will show that OFDM combined with error control coding and interleaving performs very well in FSO IM/DD systems. The key idea is to lower the symbol rate by using OFDM, and in combination with interleaving and LDPC codes obtain high tolerance to the deep fades that characterize transmission through a turbulent channel.

The third type of systems considered in this paper is long-haul fiber optics communication systems. Dominant limiting factors affecting the performance of high bit-rate return-to-zero on-off keying (RZ-OOK) systems operating at $40 \mathrm{~Gb} / \mathrm{s}$ and above are intrachannel four-wave mixing (IFWM) and intrachannel crossphase modulation (IXPM) [21]. In this case, OFDM is used to split a high-data rate data-stream into a number of low-rate datastreams that are transmitted simultaneously over a number of subcarriers. In this way, the $40 \mathrm{~Gb} / \mathrm{s}$ aggregate date rate can be divided among many subcarriers, and since per channel OFDM symbol rate is much lower, the intrachannel nonlinearities can be completely avoided. Moreover, OFDM can also be used to improve the spectral efficiency [6] of RZ-OOK, and simple equalization technique proposed in [5] can be used for chromatic dispersion compensation. We have recently shown [26] that the LDPC-coded OFDM provides very good PMD compensation, capable of differential-group delay (DGD) compensation in the order of 1500 ps and above! Notice that LDPC-coded turbo PMD equalizer proposed in [27] is suitable for DGD compensation up to 300 ps if the complexity of turbo equalizer is to be kept reasonably low.

Another possible application of LDPC-coded OFDM includes $100 \mathrm{~Gb} / \mathrm{s}$-Ethernet. Ethernet was initially introduced as a communication standard for short-distance connection among hosts in a local area networks (LAN) [16], [17]. However, due to its low-cost, high-speed, and simplicity compared to other protocols, it has been rapidly evolved, and Network Interface Cards (NICs) for 1 and $10 \mathrm{~Gb} / \mathrm{s}$ Ethernet are already commercially available [17]. Because Ethernet data rates have traditionally grown in $10 \times$ increments, data rates of $100 \mathrm{~Gb} / \mathrm{s}$ are being envisioned for the next generation [16]-[18]. Despite the recent progress in high-speed electronics, electrical time division multiplexing modulators, and photodetectors operating at $100 \mathrm{~Gb} / \mathrm{s}$ are still not widely available, so that alternative approaches to achieving a $100 \mathrm{~Gb} / \mathrm{s}$ transmission using commercially available components are of great current interest. Such an approach based on OFDM was recently proposed by our research team [8], and will be described here for the completeness of presentation.

It has been recently demonstrated experimentally (although offline) [28], [29] that both coherent and direct detection optical OFDM variants can be used for chromatic dispersion compensation, and that they are capable of compensating the residual chromatic dispersion above 77,000 ps/nm [28].
Due to the fact that all state-of-the art fiber-optic communication systems essentially use the intensity modulation/direct detection (IM/DD), we consider the LDPC-coded optical OFDM communications with direct detection only. The coherent optical OFDM systems [30], [31] require the use of an additional local laser, which increases the receiver complexity. At the same time those systems are sensitive to the laser phase noise because the OFDM symbol rate becomes comparable to the DFB laser linewidth (see [26] for more details).

The paper is organized as follows. The concept of LDPCcoded OFDM transmission is introduced in Section II. In Section III we describe several classes of LDPC codes suitable for OFDM. In Section IV LDPC-coded OFDM is discussed for possible use in POF links, while in Section $\mathrm{V}$ LDPC-coded OFMD is described for use in FSO links. In Section VI LDPC-coded OFDM is presented for use in fiber-optics communications.

\section{LDPC-CODED OFDM TRANSMISSION}

The transmitter and receiver configurations, and format of the transmitted OFDM symbol are shown in Fig. 1(a), (b), and (c), respectively. On the transmitter side an information-bearing stream at $10 \mathrm{~Gb} / \mathrm{s}$ is demultiplexed into four $2.5-\mathrm{Gb} / \mathrm{s}$ streams, which are further encoded using identical LDPC codes. The data rate employed in this step is determined by currently existing LDPC chips [19]. The outputs of these LDPC encoders are further demultiplexed and parsed into groups of $B$ bits corresponding to one OFDM frame. The $B$ bits in each OFDM frame are subdivided into $K$ subchannels with the $i$ th subcarrier carrying $b_{i}$ bits, $B=\sum b_{i}$. The $b_{i}$ bits from the $i$ th subchannel are mapped into a complex-valued signal from a $2^{b_{i}}$-point signal constellation such as QAM, which is the example constellation considered in this paper. For example, $b_{i}=2$ for QPSK and $b_{i}=4$ for 16-QAM. The complex-valued signal points from subchannels are considered to be the values of the discrete Fourier transform (DFT) of a multicarrier OFDM signal. By selecting the number of subchannels $K$, sufficiently large, OFDM symbol interval can be made significantly larger than the dispersed pulsewidth of an equivalent singlecarrier system. This spreading of the symbol interval results in significant ISI reduction. The OFDM symbol, shown in Fig. 1(c), is generated as follows: $N_{\mathrm{QAM}}(=K)$ input QAM symbols are zero-padded to obtain $N_{\mathrm{FFT}}$ input samples for IFFT, $N_{\mathrm{G}}$ nonzero samples are inserted (as explained below) to create the guard interval, and the OFDM symbol is multiplied by the window function. The purpose of cyclic extension is to preserve the orthogonality among subcarriers even when the neighboring OFDM symbols partially overlap due to dispersion, and the role of windowing is to reduce the out-of band spectrum.

The cyclic extension, illustrated in Fig. 1(c), is accomplished by repeating the last $N_{\mathrm{G}} / 2$ samples of the effective OFDM symbol part ( $N_{\mathrm{FFT}}$ samples) as a prefix, and repeating the first $N_{\mathrm{G}} / 2$ samples as a suffix. After $D / A$ conversion and $R F$ upcoversion, the $R F$ signal is mapped to the optical domain using one of the two possible options: 1) the OFDM signal directly modulates a DFB laser; or 2) the OFDM signal is used as the 


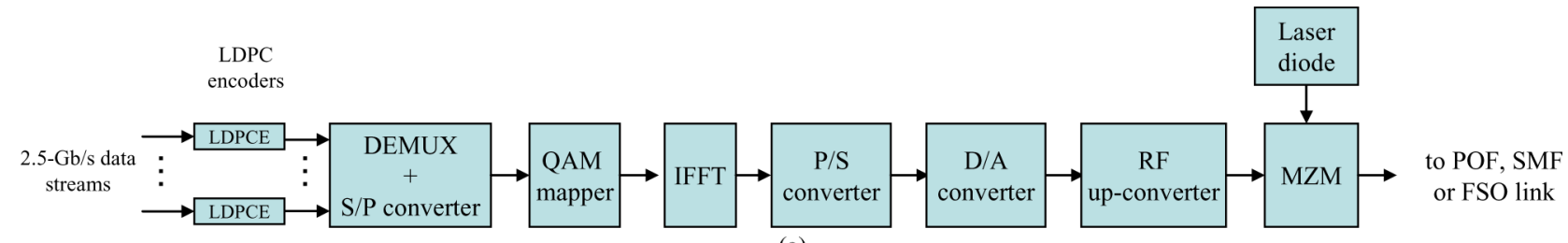

(a)

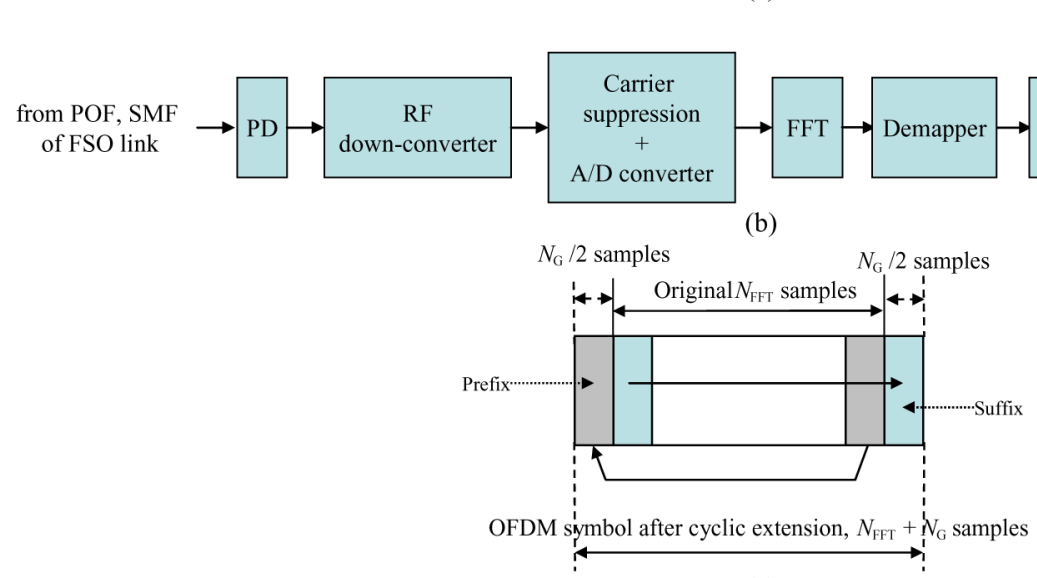

(c)

Fig. 1 (a) Transmitter configuration. (b) Receiver configuration. (c) OFDM symbol after cyclic extension. LDPCE—LDPC encoder, LDPCD—LDPC decoder, S/P—serial-to-parallel converter, MZM-Mach-Zehnder modulator, POF—plastic optical fiber, SMF—single-mode optical fiber, FSO-free-space optical link, and PD—photodetector.

RF input of a Mach-Zehnder modulator (MZM). A DC bias component is added to the OFDM signal in order to enable recovery of the QAM symbols incoherently. In what follows, three different OFDM schemes are introduced. The first scheme is based on direct modulation, and shall be referred to as the "biased-OFDM" (B-OFDM) scheme. Because bipolar signals cannot be transmitted over an IM/DD link, it is assumed that the bias component is sufficiently large so that when added to the OFDM signal the resulting sum is nonnegative. The main disadvantage of the B-OFDM scheme is its poor power efficiency. To improve OFDM power efficiency we propose two alternative schemes. The first scheme, which we shall refer to as the "clipped-OFDM" (C-OFDM) scheme, is based on single-side band (SSB) transmission, and clipping of the OFDM signal after bias addition. The bias is varied in order to find the optimum one for fixed optical launched power. It was found that the optimum bias is one in which $\sim 50 \%$ of the total electrical signal energy before clipping is allocated for transmission of a carrier. The second power-efficient scheme, which we shall refer to as the "unclipped-OFDM" (U-OFDM) scheme, is based on SSB transmission using a $\mathrm{LiNbO}_{3} \mathrm{MZM}$. To avoid distortion due to clipping at the transmitter, the information is mapped into the optical domain by modulating the electrical field of the optical carrier (instead of intensity modulation employed in the B-OFDM and C-OFDM schemes). In this way, both positive and negative portions of the electrical OFDM signal are transmitted to the photodetector. Distortion introduced by the photodetector, caused by squaring, is successfully eliminated by proper filtering, and recovered signal does not exhibit significant distortion. It is important to note, however, that the U-OFDM scheme is slightly less power efficient that the C-OFDM scheme.
High-speed optical receivers commonly employ the transimpedance design, which is a good compromise between noise and bandwidth. A preamplified PIN photodiode or an avalanche photodiode is typically used as an optical detector. The PIN photodiode output current can be written as

$$
\begin{aligned}
i(t)= & \mathrm{R}\left|\left(s_{\mathrm{OFDM}}(t)+b\right) * h(t)\right|^{2}=\mathrm{R}\left[\left|s_{\mathrm{OFDM}}(t) * h(t)\right|^{2}\right. \\
& \left.+|b * h(t)|^{2}+2 R_{e}\left\{\left(s_{\mathrm{OFDM}}(t) * h(t)\right)(b * h(t))\right\}\right]
\end{aligned}
$$

where $s_{\text {OFDM }}(t)$ denotes the transmitted OFDM signal in $R F$ domain, upon $D / A$ conversion and $R F$ up-conversion, defined by

$$
\begin{gathered}
s_{\mathrm{OFDM}}(t)=R e\left\{\sum_{k=-\infty}^{\infty} w(t-k T) \sum_{i=-\left(N_{\mathrm{FFT}} / 2\right)}^{\left(N_{\mathrm{FFT}} / 2\right)-1}\right. \\
\left.\times X_{i, k} e^{j 2 \pi \frac{i}{T_{\mathrm{FFT}}} \cdot(t-k T)} e^{j 2 \pi f_{R F t}}\right\} \\
k T-T_{\mathrm{G}} / 2-T_{\mathrm{win}} \leq t \leq k T+T_{\mathrm{FFT}}+T_{\mathrm{G}} / 2+T_{\mathrm{win}}
\end{gathered}
$$

where $b$ is the DC bias component, and $R$ denotes the photodiode responsivity. In (2) $X_{i, k}$ denotes the $i$ th subcarrier QAM symbol of the $k$ th OFDM symbol, $T$ denotes the OFDM symbol duration, $T_{\mathrm{FFT}}$ is the FFT part duration, $T_{\mathrm{G}}$ is the guard interval (cyclic extension) duration, $T_{\text {win }}$ denotes the windowing interval duration, $w(t)$ is the window function, and $f_{\mathrm{RF}}$ denotes the $\mathrm{RF}$ carrier frequency. The impulse response of the optical channel is represented by $h(t)$. We treat several different transmission media in this paper: 1) GI-POF: it was found in [9], [10] that 
GI-POF can be modeled as a Gaussian filter; 2) multimode fiber: the transfer function of a multimode fiber links is presented in [22]; 3) FSO links: the details of our FSO link model are described in Section V; and 4) long-haul fiber links: we modeled this channel by solving the nonlinear Schrödinger equation numerically using split-step Fourier method as described in [23]. The signal after $R F$ down-conversion and appropriate filtering, can be written as

$$
r(t)=\left[i(t) k_{R F} \cos \left(\omega_{R F^{t}}\right)\right] * h_{e}(\tau)+n(t)
$$

where $h_{e}(t)$ is the impulse response of the low-pass filter (having the transfer function $\left.H_{\mathrm{e}}(\mathrm{j} \omega)\right), n(t)$ is electronic noise in the receiver, and $k_{\mathrm{RF}}$ denotes the $R F$ down-conversion coefficient. Finally, after the $A / D$ conversion and cyclic extension removal, the signal is demodulated by using the FFT algorithm. The soft outputs of the FFT demodulator are used to estimate the bit reliabilities that are fed to identical LDPC iterative decoders implemented based on the sum-product algorithm [12].

\section{SElEction OF LDPC CODES}

Now we turn our attention to the selection of LDPC codes. Three classes of FEC based on girth-8 LDPC codes are introduced in this section (girth refers to the shortest cycle length in corresponding bipartite graph of an LDPC code [12]). The first code-class is the class of girth-8 regular LDPC codes designed based on the concept of mutually-orthogonal Latin rectangles (MOLS) [20]. The LDPC codes designed using the concept of MOLS naturally arise as girth-6 codes, and to increase the girth up to 8 we employed an iterative algorithm we originally described in [24]. The second code-class is the class of irregular girth-8 LDPC codes obtained from the previous class by selective inverting certain ones in the parity-check matrix into zeros (i.e., those with column weights 3, 4, and 5). The third-class of codes is class of an array code of girth- 8 , which can be described as follows:

$$
H=\left[\begin{array}{ccccc}
P^{i_{1}} & P^{i_{2}} & P^{i_{3}} & \ldots & P^{i_{q}} \\
P^{i_{q}} & P^{i_{1}} & P^{i_{2}} & \ldots & P^{i_{q-1}} \\
\ldots & \ldots & \ldots & \ldots & \ldots \\
P^{i_{q-r+2}} & P^{i_{q-r+3}} & P^{i_{q-r}+4} & \ldots & P^{i_{q-r+1}}
\end{array}\right]
$$

with $P$ being the permutation matrix $P=\left(p_{i j}\right)_{n x n}, p_{i, i+1}=$ $p_{n, 1}=1$ (zero otherwise). The exponents $i_{1}, i_{2} \ldots i_{q}$ in (4) are carefully chosen to avoid the cycles of length six in corresponding bipartite graph of a parity-check matrix. In [25] we have shown that when exponents in are selected as elements from the following set

$$
L=\left\{i: 0 \leq i \leq p^{2}-1, \quad \theta^{i}+\theta \in G F(p)\right\}
$$

where $p$ is a prime and $\theta$ is the primitive element of the finite field $G F\left(p^{2}\right)$; the resulting LDPC code is of girth-6. To increase the girth, we carefully converted some of the permutation blocks in (4) into all-zeros blocks.

Given this general description of LDPC-coded OFDM and our selection of LDPC codes we turn our attention now to the four applications of interests.

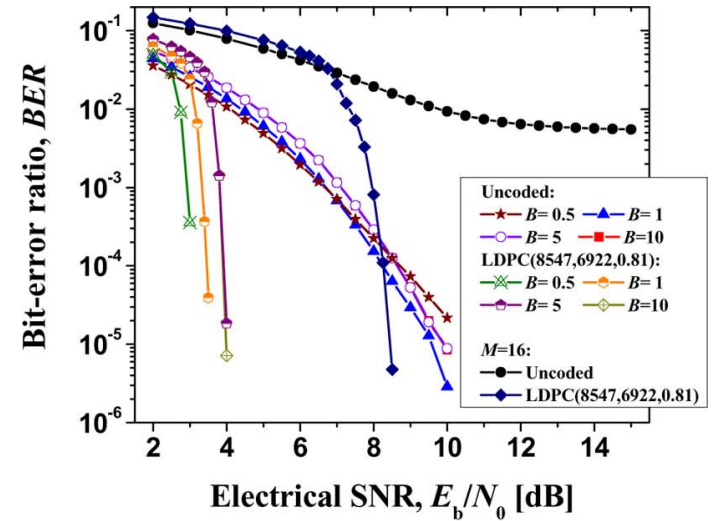

(a)

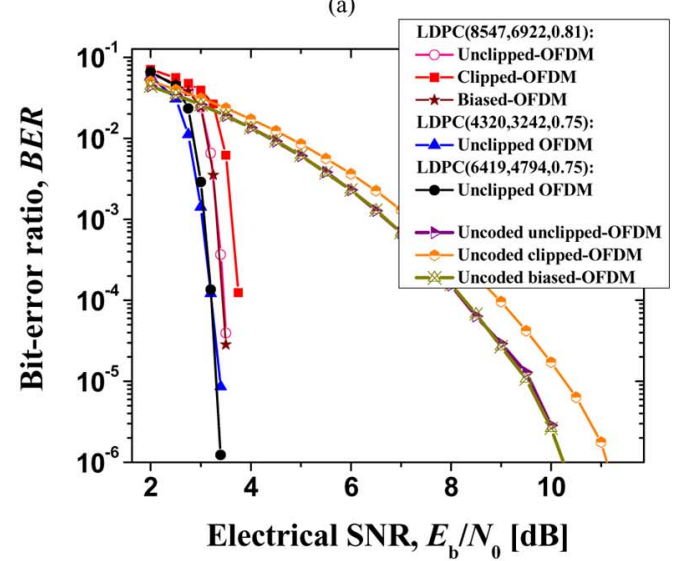

(b)

Fig. 2 BER performance of LDPC-coded OFDM for transmission over GIPOF links. (a) Comparison for different normalized bandwidths. (b) Comparison of different LDPC codes and different OFDM schemes.

\section{LDPC-CODED OFDM OVER GI-POF LINKS}

The first application of interest described here is LDPC-coded OFDM over the GI-POF channel. For normalized bandwidths $B \geq 1$ [the bandwidth is normalized with respect to the aggregate bit rate $(10-\mathrm{Gb} / \mathrm{s})]$, the OFDM system parameters are selected as follows. The total OFDM signal bandwidth is set to $0.25 \mathrm{~B}$, the number of subchannels is set to $N_{\mathrm{QAM}}=64$, the required FFT/IFFT are calculated using $N_{\mathrm{FFT}}=128$ points, the $R F$ carrier frequency is set to $0.75 B$, the bandwidth of the optical filter for SSB transmission is set to $2 B$, and the total average optical launched power is set to $0 \mathrm{dBm}$. The guard interval is obtained by cyclic extension using $N_{\mathrm{G}}=2 \times 16$ samples as explained earlier. For normalized bandwidth $B<1$ all parameters remain the same except for the $R F$ carrier frequency that is set to $0.3 B$. The results of simulations are shown in Fig. 2 for different values of GI-POF normalized bandwidth $B$.

Three classes of LDPC codes, as explained in the previous section, are considered in simulations. The first class is the girth- 8 regular LDPC code $(8547,6922)$ of rate 0.81 . The second class is irregular girth-8 LDPC code $(6419,4794)$ of rate 0.75 . The third class is girth- 8 regular block-circulant LDPC code $(4320,3242)$ of rate 0.75 designed as explained in Section III [see eq. (4)]. In the simulations shown in Fig. 2(a) the LDPC code of rate 0.81 is employed, and bit-error rate (BER) 
results for different GI-POF normalized bandwidths are reported for the U-OFDM scheme. For normalized bandwidth $B=0.5$ and QPSK U-OFDM, the LDPC code of rate 0.81 provides a coding gain of approximately $9 \mathrm{~dB}$ at BER of $10^{-6}$. Much larger coding gain is expected at smaller values of BER. The uncoded 16-QAM U-OFDM scheme exhibits a BER floor, while the LDPC-coded OFDM scheme is able to operate error-free. Notice that alternative FEC schemes based on RS codes [such as $\mathrm{RS}(255,239)$ ] or a concatenation of two RS-codes (such as $\mathrm{RS}(255,239)+\mathrm{RS}(255,223))$ are unable to operate for 16-QAMOFDM because the error floor of the uncoded signal is too high for their error correction capabilities. In Fig. 2(b) different classes of LDPC codes are compared for QPSK U-OFDM, and it is found that the irregular LDPC code of rate 0.75 outperforms the other two classes of LDPC codes. In the same Figure, the LDPC-coded B-OFDM, U-OFDM, and C-OFDM schemes are compared in terms of BER. It is found that B-OFDM and $\mathrm{U}-\mathrm{OFDM}$ schemes perform comparable, while the C-OFDM is about $0.5 \mathrm{~dB}$ worse. Notice, however, that C-OFDM is the most power-efficient, whereas B-OFDM is the worst in that sense.

\section{LDPC-CODED OFDM OVER FSO LINKS}

The second application of interest is LDPC-coded OFDM over FSO channel. The point-to-point FSO system considered here, shown in Fig. 3, consists of an FSO-OFDM transmitter, a turbulent propagation medium and an FSO-OFDM receiver. The modulated beam is projected toward the receiver using an expanding telescope. At the receiver, an optical system collects the light, and focuses it onto a detector, which delivers an electrical signal proportional to the incoming optical power. Notice that no aperture averaging is applied. Once again we employ a transimpedance receiver.

For the sake of illustration, the signal waveforms and powerspectral densities (PSDs) at different points in the OFDM system in a back-to-back configuration (without any propagation model) are given in Fig. 4 for SSB transmission. The bandwidth of the OFDM signal is set to $2.5 \mathrm{GHz}$, and the RF carrier to $7.5 \mathrm{GHz}$. The number of OFDM subchannels is set to 64 . The OFDM sequence is zero-padded and the FFT is calculated once again using 128 points. The guard interval is obtained by a cyclic extension of $2 \times 16$ samples as explained above. The windowing $(2 \times 16$ samples $)$ is based on the Blackman-Harris windowing function. The average transmitted optical power is set to $0 \mathrm{dBm}$. To avoid distortions due to RF driver amplifier saturation and MZM nonlinear transfer function, the OFDM system is designed in such a way that both the RF driver amplifier and MZM operate in their linear regime [see Figs. 4(a)-(c)] The PSD for SSB OFDM MZM output signal is shown in Fig. 4(d), and the photodetector output signal (for SSB OFDM transmission) is shown in Fig. 4(e).

A common turbulence model assumes that the variations in medium temperature and pressure due to solar heating and wind can be understood as individual cells of air or eddies of different diameters and refractive indices [15]. These eddies may be considered as lenses randomly refracting the optical wave front, and producing a distorted intensity profile at the receiver side of

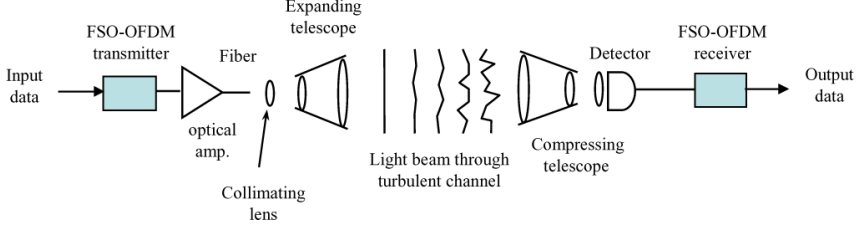

Fig. 3 Free-space optical communication link under consideration.

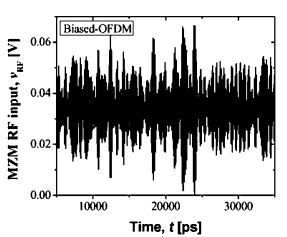

(a)

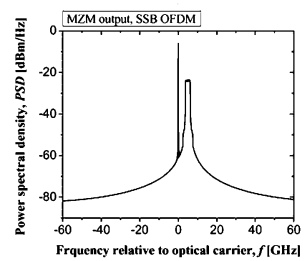

(d)

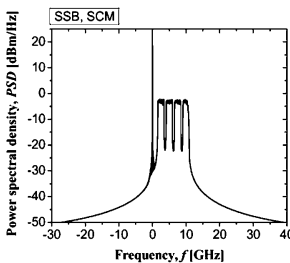

(g)

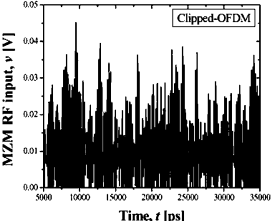

(b)

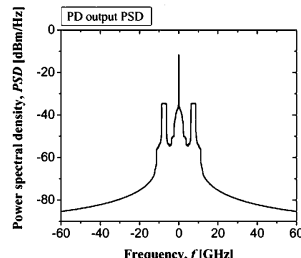

(e)

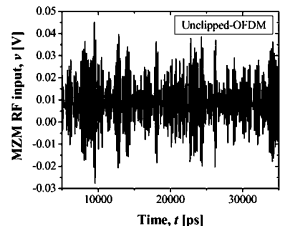

(c)

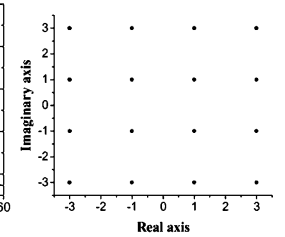

(f)

Waveforms and power spectral densities of the SSB OFDM signal with 64 subcarriers at different points during transmission of an OFDM signal in a back-to-back configuration. (a) MZM RF input for B-OFDM. (b) MZM RF input for C-OFDM. (c) MZM RF input for U-OFDM. (d) PSD after MZM (U-OFDM). (e) Photodectector (PD) output PSD (U-OFDM). (f) Receiver constellation diagram for 16-QAM (U-OFDM). (g) PSD of SCM signal with four OFDM channels (U-OFDM). The PSD of double-side band OFDM signal after MZM is shown in Fig. 2 (h) (U-OFDM).

an FSO communication link. The most widely accepted theory of turbulence is due to [15], and it assumes that kinetic energy from large turbulent eddies, characterized by the outer scale $L_{0}$, is transferred without loss to eddies of decreasing size down to sizes of a few millimeters characterized by the inner scale $l_{0}$. The inner scale represents the cell size at which energy is dissipated by viscosity. The refractive index varies randomly across the different turbulent eddies, and causes phase and amplitude variations to a propagating optical wave front. Turbulence can also cause random drifts of optical beams-a phenomenon usually referred to as wandering - and can induce beam focusing. To quantify the strength of the turbulence we use the unitless Rytov variance, given by [15]

$$
\sigma_{R}^{2}=1.23 C_{n}^{2} k^{7 / 6} L^{11 / 6}
$$

where $k=2 \pi \lambda$ is the optical wave number, $\lambda$ is the wavelength, $L$ is propagation distance, and $C_{n}^{2}$ is the refractive index structure parameter, which we assume to be constant for 


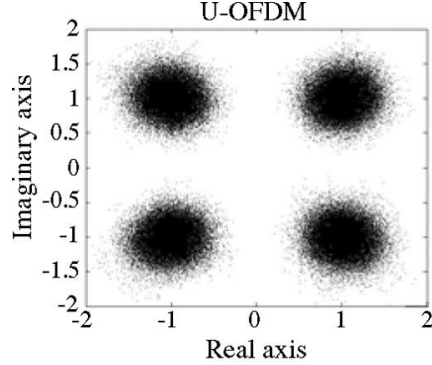

(a)

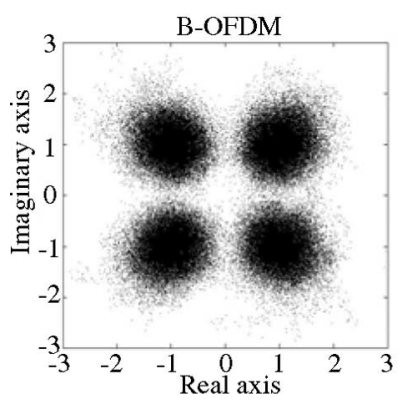

(c)

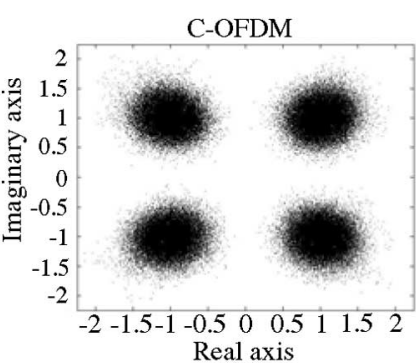

(b)

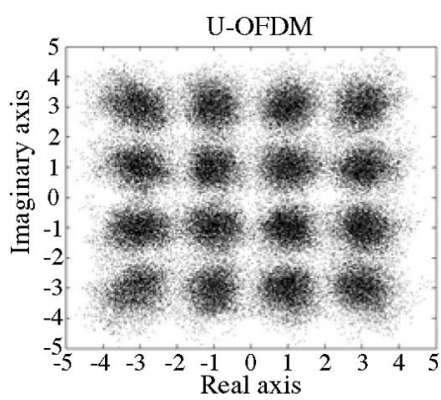

(d)
Fig. 5 Received constellation diagrams of QPSK (a)-(c) and 16-QAM (d) SSB FSO-OFDM systems with electrical SNR per bit of $18 \mathrm{~dB}$ under the weak turbulence $\left(\sigma_{R}=0.6\right)$ for (a),(d) U-OFDM scheme. (b) C-OFDM scheme. (c) B-OFDM scheme.

horizontal paths. Although the Rytov variance represents the scintillation index of an unbounded plane wave in the weak turbulence regime, it can also be used as an intuitive metric that brings together all the physical parameters.

The influence of both the atmospheric turbulence and electronic noise on QPSK and 16-QAM SSB FSO-OFDM systems is illustrated in Fig. 5. A SSB OFDM system with 64 subcarriers is used. The average launched power is once again set to $0 \mathrm{dBm}$, the electrical signal-to-noise ratio is set to $18 \mathrm{~dB}$, and all received signal constellation diagrams are obtained assuming weak atmospheric turbulence $\left(\sigma_{R}=0.6\right)$. The atmospheric turbulence changes the symmetry of clusters from circular for AWGN channel to elliptic (see Fig. 5). Both C-OFDM and U-OFDM schemes are more immune to the atmospheric turbulence than B-OFDM scheme. The U-OFDM system is only slightly more immune to the atmospheric turbulence than C-OFDM scheme. It appears that the better power efficiency of C-OFDM compensates the distortion introduced by clipping. Namely, the average launched power is fixed for all three OFDM schemes implying that more energy per bit is allocated in the C-OFDM scheme (because the power in DC bias is lower), and as a consequence the scheme is more immune to electrical noise. On the other hand, U-OFDM requires appropriate rejection filters to deal with photodetector nonlinearity. Higher immunity to electrical noise and imperfections in U-OFDM rejection filter may result in slightly better BER performance of C-OFDM scheme when compared to UOFDM scheme (see Fig. 6).

Simulation results of an LDPC coded SSB U-OFDM system for two different turbulence strengths are given in Fig. 6. For BPSK and QPSK, the coding gain improvement of an LDPC encoded FSO-OFDM system over an LDPC encoded OOK FSO

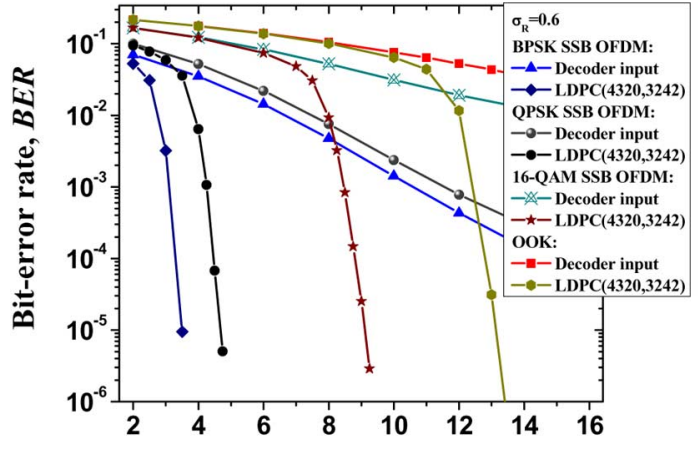

Electrical signal-to-noise ratio, $E_{\mathrm{b}} / N_{0}[\mathrm{~dB}]$

(a)

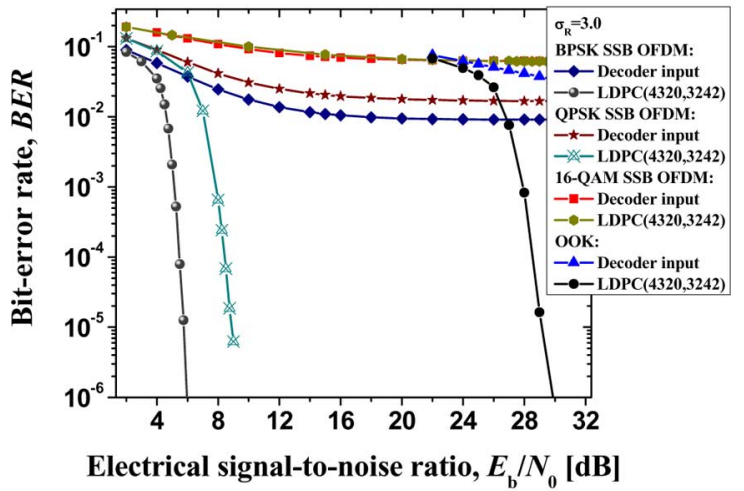

(b)

Fig. 6 BER performance of LDPC-coded SSB U-OFDM system with 64subcarriers under (a) Weak turbulence $\left(\sigma_{R}=0.6\right)$. (b) Strong turbulence $\left(\sigma_{R}=3.0\right)$.

system increases as the turbulence strength increases. However, the 16-QAM FSO-OFDM system is not able to operate in the regime of strong turbulence. For weak turbulence $\left(\sigma_{R}=0.6\right)$ [see Fig. 6(a)] the coding gain improvement of LDPC coded FSO-OFDM system with 64 subcarriers over the LDPC encoded FSO OOK system is $8.47 \mathrm{~dB}$ for QPSK and $9.66 \mathrm{~dB}$ for BPSK, at the BER of $10^{-5}$.

For strong turbulence $\left(\sigma_{R}=3.0\right)$ [see Fig. 6(b)] the coding gain improvement of the LDPC coded FSO-OFDM system over the LDPC coded FSO OOK system is $20.24 \mathrm{~dB}$ for QPSK and $23.38 \mathrm{~dB}$ for BPSK. In both cases block-circulant LDPC code $(4320,3242)$ of rate 0.75 is employed.

The comparison of different LDPC coded SSB OFDM schemes in weak turbulence $\left(\sigma_{R}=0.6\right)$, is given in Fig. 7 . C-OFDM scheme slightly outperforms the U-OFDM scheme. Both C-OFDM and U-OFDM schemes outperform the BOFDM scheme by approximately $1.5 \mathrm{~dB}$ at BER of $10^{-5}$.

We are turning our attention now to the LDPC-coded OFDM applications in fiber-optics communications.

\section{LDPC-CODED OFDM FIBER-OPTICS COMMUNICATIONS}

The main reasons for suitability of the OFDM for long-haul transmission are [6]: 1) improvement of spectral efficiency; 2) simplification of the chromatic dispersion compensation engineering; 3 ) the possibility of avoiding the intrachannel nonlinearities because the OFDM symbol rate is much lower than 


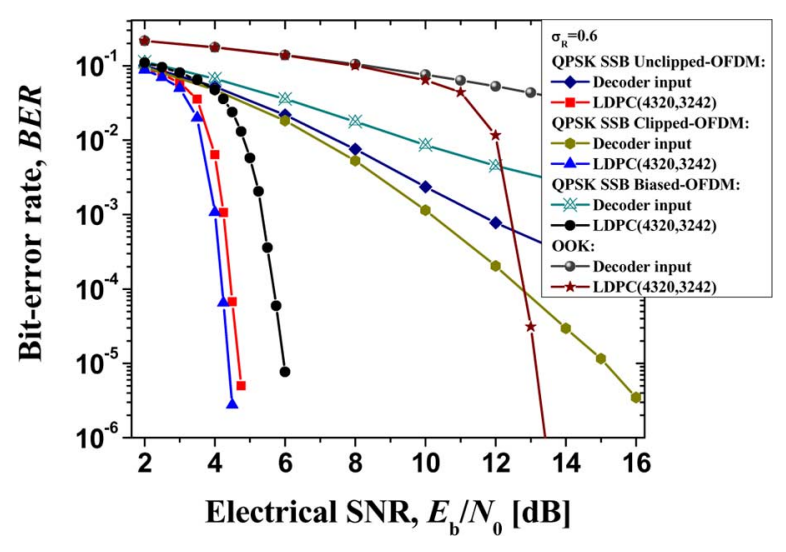

Fig. 7 Comparison of different LDPC coded SSB FSO-OFDM systems with 64 -subcarriers under the weak turbulence $\left(\sigma_{R}=0.6\right)$.

aggregate data rate; and 4) PMD compensation [26]. The BER performance of the LDPC-coded U-OFDM scheme (with aggregate data rate $40 \mathrm{~Gb} / \mathrm{s}$ ) against conventional LDPC-coded RZ-OOK scheme (operating at $40 \mathrm{~Gb} / \mathrm{s}$ ) is given in Fig. 8 for the linear channel model. The LDPC-coded QPSK U-OFDM provides more than $2 \mathrm{~dB}$ coding gain improvement over LDPC coded RZ-OOK at BER of $10^{-8}$. We have shown in [6] that LDPC-coded OFDM provides much higher spectral efficiency than LDPC-coded RZ-OOK.

Another possible application in fiber-optics communications is $100 \mathrm{~Gb} / \mathrm{s}$-Ethernet [8]. For example, for QPSK OFDM transmission two $1 \mathrm{~Gb} / \mathrm{s}$-streams create a QPSK signal constellation point; with 50 subcarriers carrying $2 \mathrm{~Gb} / \mathrm{s}$ traffic, the aggregate rate of $100 \mathrm{~Gb} / \mathrm{s}$ can be achieved. The BER curves for the uncoded $100 \mathrm{~Gb} / \mathrm{s}$ OFDM SSB transmission using QPSK are shown in Fig. 9 for dispersion map described in [8]. As already mentioned in Section II we modeled the long-haul fiber-optics channel by solving the nonlinear Schrödinger equation numerically using split-step Fourier method [23]. It is evident that $100 \mathrm{~Gb} / \mathrm{s}$ transmission over $3840 \mathrm{~km}$ is possible using OFDM and LDPC codes with threshold BER of $10^{-2}$ (for launched power of $-3 \mathrm{dBm}$ ). For more details an interesting reader is referred to our recent article [8].

In the presence of PMD, the PIN photodiode output current can be written as follows:

$$
\begin{aligned}
i(t)= & R\left\{\left|\sqrt{k}\left(s_{\mathrm{OFDM}}(t)+b\right)^{*} h_{V}(t)\right|^{2}\right. \\
& \left.+\left|\sqrt{1-k}\left(s_{\mathrm{OFDM}}(t)+b\right)^{*} h_{H}(t)\right|^{2}\right\}
\end{aligned}
$$

where $k$ denotes the power-splitting ratio between two principal states of polarizations (PSPs). For the first order PMD, the optical channel responses $\left.h_{\mathrm{H}} t\right)$ and $h_{\mathrm{V}}(t)$ of horizontal and vertical PSPs are given as [26] $h_{\mathrm{H}}(t)=\delta(t+\Delta \tau / 2)$ and $h_{\mathrm{V}}(t)=\delta(t-\Delta \tau / 2)$, respectively, where $\Delta \tau$ is the DGD of two PSPs. From [26], it follows that in the presence of the first order PMD, the photodiode output signal, after appropriate filtering to remove the squared and DC terms, is proportional to

$$
\begin{aligned}
i(t) \sim & 2 R b\left[k \cdot s_{\mathrm{OFDM}}(t-\Delta \tau / 2)\right. \\
& \left.+(1-k) \cdot s_{\mathrm{OFDM}}(t+\Delta \tau / 2)\right] .
\end{aligned}
$$

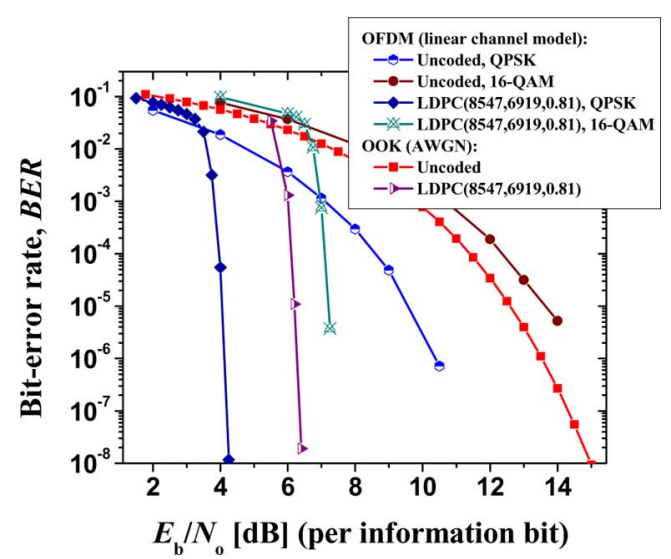

Fig. 8 BER performance of OFDM against OOK for a linear channel model.

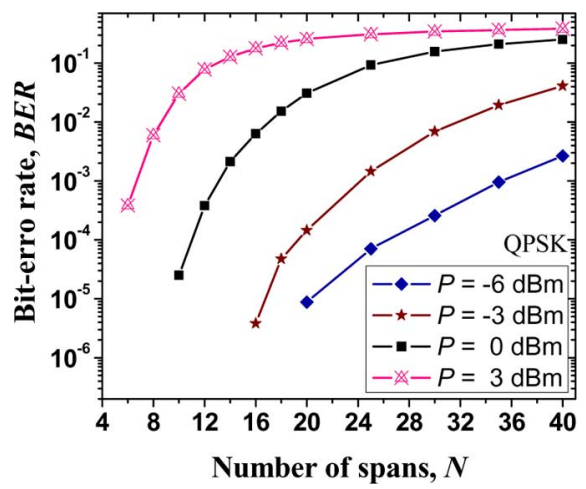

Fig. 9 Uncoded BER versus number of spans for SSB QPSK OFDM transmission for dispersion map from [8].

Equation (7) is reminiscent to a two-ray multipath wireless model [1], and therefore the channel estimation techniques similar to those employed in wireless communications are straightforwardly applicable here. For example, simple channel estimation technique [26] based on short training sequence can be used to compensate for DGD in excess of $1500 \mathrm{ps}$. The signal constellation diagrams, after the demapper from Fig. 1, and before and after applying the channel estimation are shown in Fig. 10(a) and (b). They correspond to the worst case scenario $(k=1 / 2)$ and $10 \mathrm{~Gb} / \mathrm{s}$ aggregate data rate. Therefore, the channel estimation based OFMD is able to compensate for DGD of 1600 ps. The similar channel estimation technique can be used to compensate for SPM [6], [8]. For example, the signal constellation diagrams before and after SPM phase correction are shown in Fig. 11(a) and (b) for transmission distance of $1200 \mathrm{~km}$, for dispersion map described in [8] and aggregate data rate of $100 \mathrm{~Gb} / \mathrm{s}$. The four pilot tones were sufficient to cancel the phase rotation due to SPM. The same equalization scheme can be used for intermodal dispersion compensation [7] and chromatic dispersion compensation [7], [28].

\section{SUMMARY}

We discussed LDPC-coded OFDM as a universal coded modulation technique suitable for use in variety of optical channels: 1) plastic optical fiber; 2) atmospheric turbulent channel; and 3) 


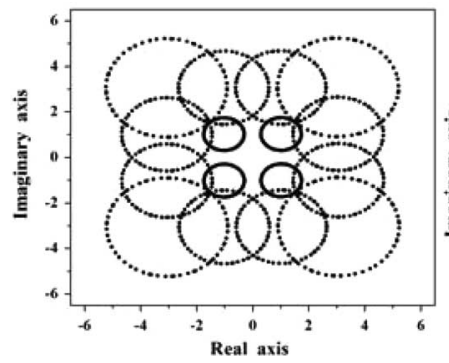

(a)

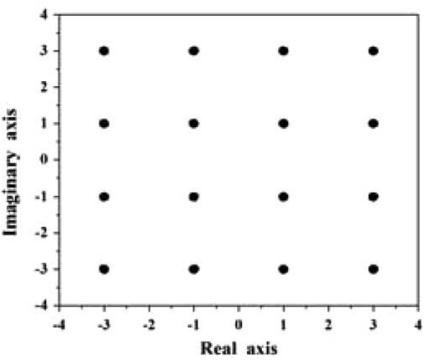

(b)
Fig. 10 (a) Signal constellation diagram before PMD compensation for DGD of 1600 ps. (b) Signal constellation diagram after PMD compensation (other effects except PMD have been ignored).

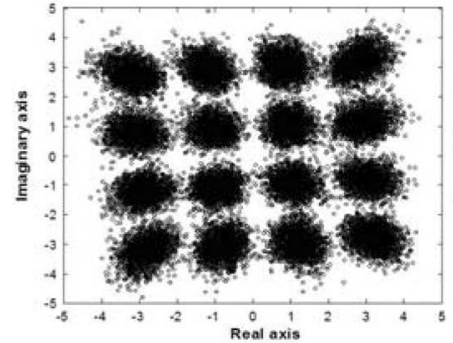

(a)

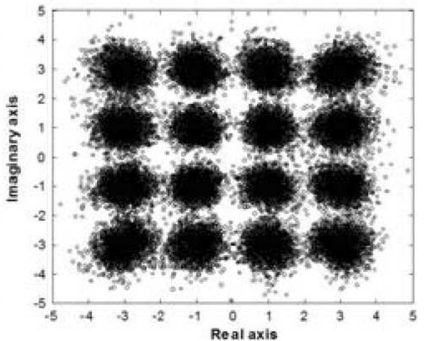

(b)
Fig. 11 Received signal constellation for 16-QAM SSB transmission after $1200 \mathrm{~km}$ for dispersion map from [8], and aggregate data rate of $100-\mathrm{Gb} / \mathrm{s}$. (a) Before SPM phase correction. (b) After the SPM phase correction.

fiber-optics channel. Moreover, LDPC-coded OFDM was considered in context of $100 \mathrm{~Gb} / \mathrm{s}$-Ethernet. Due to severe dispersion distortion of signals at $10 \mathrm{~Gb} / \mathrm{s}$ and above transmitted over GI-POFs or severe distortion due to atmospheric turbulence, a powerful forward FEC schemes much more powerful than those already proposed for use in long-haul transmission are required. Several such FEC schemes based on girth-8 LDPC codes are described. We have shown that LDPC-coded OFDM is able to operate even in the regime when standard FEC schemes such as RS and concatenated RS codes, are overwhelmed with errors, and cannot provide error free transmission at all. We have shown in Section V that LDPC-coded OFDM can enable the transmission under strong atmospheric turbulence. The OFDM also provides good PMD compensation capable of compensating DGD of 1600 ps. The pilot based channel estimation can be used to deal with fiber nonlinearities.

\section{REFERENCES}

[1] R. Prasad, OFDM for Wireless Communications Systems. Boston, London: Artech House, 2004.

[2] Y. Wu and B. Caron, "Digital television terrestrial broadcasting," IEEE Commun. Mag., vol. 32, no. 5, pp. 46-52, May 1994.

[3] A. Kim et al., "60 GHz wireless communication systems with radiooverfiber links for indoor wireless LANs," IEEE Trans. Consum. Electron., vol. 50, no. 2, pp. 517-520, May 2004.

[4] I. B. Djordjevic, B. Vasic, and M. A. Neifeld, "LDPC coded orthogonal frequency division multiplexing over the atmospheric turbulence channel," in Proc. CLEO/QELS 2006, Paper CMDD5, Long Beach, CA, May 21-26.

[5] A. J. Lowery, L. Du, and J. Armstrong, "Orthogonal frequency division multiplexing for adaptive dispersion compensation in long haul WDM systems," in Proc. OFC Postdeadline Papers, Paper no. PDP39, Anaheim, CA, Mar. 2006.

[6] I. B. Djordjevic and B. Vasic, "Orthogonal frequency-division multiplexing for high-speed optical transmission," Opt. Express, vol. 14, pp. 37673775, May 1, 2006.

[7] A. J. Lowery and J. Armstrong, "10 Gb/s multimode fiber link using power-efficient orthogonal-frequency-division multiplexing," Opt. Express, vol. 13, no. 25, p. 10003, Dec. 12, 2005.

[8] I. B. Djordjevic and B. Vasic, " $100 \mathrm{~Gb} / \mathrm{s}$ transmission using orthogonal frequency-division multiplexing," IEEE Photon. Technol. Lett., vol. 18, no. 15 , pp. 1576-1578, Aug. 2006.

[9] B.-G. Shin, J.-H. Park, and J.-J. Kim, "Low-loss, high-bandwidth gradedindex plastic optical fiber fabricated by the centrifugal deposition method," App. Phys. Lett., vol. 82, no. 26, pp. 4645-4647, Jun. 30, 2003.

[10] M. Sibley, "Analysis of multiple pulse position modulation when operating over graded-index plastic optical fibre," in Proc. Inst. Electr. Eng. Optoelectron., vol. 151, Dec. 2005, pp. 469-475.

[11] S. Randel et al., "1 Gbit/s transmission with $6.3 \mathrm{bits} / \mathrm{s} / \mathrm{Hz}$ spectral efficiency in a $100 \mathrm{~m}$ standard $1 \mathrm{~mm}$ step-index plastic optical fibre link using adaptive multiple subcarrier modulation," in Proc. Post deadline papers ECOC 2006, Paper Th4.4.1, Cannes, France, Sep. 24-28,

[12] I. B. Djordjevic et al., "Low-density parity-check codes for $40 \mathrm{~Gb} / \mathrm{s}$ optical transmission systems," IEEE J. Sel. Topics Quantum Electron., vol. 12 no. 4, pp. 555-562, Jul./Aug. 2006.

[13] X. Zhu and J.M. Kahn, "Free-space optical communication through atmospheric turbulence channels," IEEE Trans. Commun., vol. 50, no. 8, pp. 1293-1300, Aug. 2002.

[14] M. A. Al-Habash, L. C. Andrews, and R. L. Phillips, "Mathematical mode for the irradiance probability density function of a laser beam propagating through turbulent media," Opt. Eng., vol. 40, pp. 1554-1562, 2001.

[15] L. C. Andrews and R. L. Philips, Laser Beam Propagation Through Random Media. Bellingham, WA: SPIE Opt. Eng. Press, 1998.

[16] A. Zapata et al., "Next-generation 100-gigabit metro ethernet (100 GbME) using multiwavelength optical rings," J. Lightw. Technol., vol. 22, pp. 2420-2434, Nov. 2004.

[17] M. Duelk, "Next generation 100 G Ethernet," in Proc ECOC 2005, Paper no. Tu3.1.2. Glasgow, Scotland, U.K, pp. 25-29.

[18] G. Raybon, P. J. Winzer, and C. R. Doerr, " $10 \times 107-\mathrm{Gb} / \mathrm{s}$ electronically multiplexed and optically equalized NRZ transmission over $400 \mathrm{~km}$," in Proc. OFC Postdeadline Papers, Paper no. PDP32, Anaheim, CA, Mar. 2006.

[19] C.-C. Lin, K.-L. Lin, H.-C. Chang, and C.-Y. Lee, "A 3.33 Gb/s (1200,720) low-density parity check code decoder," in Proc. ESSCIRC 2005, Grenoble, France, 2005, pp. 211-214.

[20] I. B. Djordjevic and B. Vasic, "MacNeish-Mann theorem based iteratively decodable codes for optical communication systems," IEEE Commun. Lett., vol. 8, no. 8, pp. 538-540, Aug. 2004.

[21] I. B. Djordjevic and B. Vasic, "Constrained coding techniques for the suppression of intrachannel nonlinear effects in high-speed optical transmission," IEEE J. Lightw. Technol., vol. 24, no. 1, pp. 411-419, Jan. 2006.

[22] I. Gasulla and J. Campany, "Transfer function of multimode fiber links using an electric filed propagation model: Application to radio over fiber systems," Opt. Express, vol. 14, no. 20, p. 9051.

[23] G. P. Agrawal, Nonlinear Fiber Optics. San Diego, CA: Academic, 2001.

[24] B. Vasic, I. B. Djordjevic, and R. Kostuk, "Low-density parity check codes and iterative decoding for long haul optical communication systems," IEEE/OSA J. Lightw. Technol., vol. 21, no. 2, Feb. 2003, pp. 438-446.

[25] O. Milenkovic, I. B. Djordjevic, and B. Vasic, "Block-circulant lowdensity parity-check codes for optical communication systems," IEEE J. Sel. Top. Quantum Electron., vol. 10, no. 2, pp. 294-299, Mar./Apr. 2004.

[26] I. B. Djordjevic, "PMD compensation in fiber-optic communication systems with direct detection using LDPC-coded OFDM," Opt. Express, vol. 15, pp. 3692-3701, 2007.

[27] I. B. Djordjevic, H. G. Batshon, M. Cvijetic, L. Xu, and T. Wang, "PMD compensation by LDPC-coded turbo equalization," IEEE Photon. Technol. Lett., vol. 19, no. 15, pp. 1163-1165, Aug. 2007.

[28] S. L. Jansen, I. Morita, N. Takeda, and H. Tanaka, "20-Gb/s OFDM transmission over 4,160-km SSMF enabled by RF-pilot tone phase compensation," in Proc. OFC/ NFOEC 2007 Postdeadline Papers, Paper no. PDP15, Anaheim, CA, Mar. 25-29.

[29] B. J. Schmidt, A. J. Lawery, and J. Amstrong, "Experimental demonstration of $20 \mathrm{Gbit} / \mathrm{s}$ direct-detection optical OFDM and $12 \mathrm{Gbit} / \mathrm{s}$ with a 
colorless transmitter," in OFC/ NFOEC 2007 Postdeadline Papers, Paper no. PDP18, Anaheim, CA, Mar. 25-29.

[30] W. Shieh and C. Athaudage, "Coherent optical frequency division multiplexing," Electron. Lett., vol. 42, pp. 587-589, 2006.

[31] W. Shieh, "PMD-supported coherent optical OFDM systems," IEEE Photon. Technol. Lett., vol. 19, no. 3, pp. 134-136, Feb. 2007.

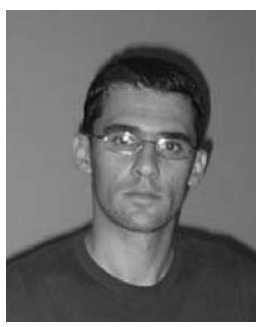

Ivan B. Djordjevic (M’04) received the B.Sc., M.Sc., and $\mathrm{Ph} . \mathrm{D}$. degrees in electrical engineering from the University of Nis, Nis, Serbia, in 1994, 1997, and 1999, respectively.

$\mathrm{He}$ is an Assistant Professor of electrical and computer engineering at the University of Arizona, Tucson. Prior to this appointment in August 2006, he was with the University of the West of England, Bristol, UK; University of Bristol, UK; Tyco Telecommunications, Eatontown, USA; and National Technical University of Athens, Athens, Greece. His current research interests include optical communication systems, error control coding, constrained coding, coded modulation, turbo equalization, and OFDM applications. He is the author or coauthor of more than 100 international published papers.

Dr. Djordjevic is a director of the Optical Communication Systems Laboratory (OCSL) within the ECE Department at the University of Arizona.

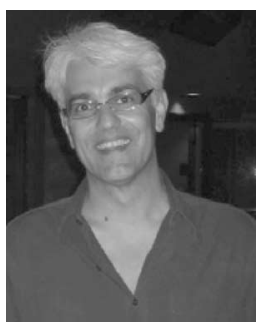

Bane Vasic (S'92-M'93-SM'02) received the B.Sc., M.Sc., and Ph.D. degrees in electrical engineering from the University of Nis, Nis, Serbia, in 1989, 1991, and 1994, respectively.

From 1996 to 1997, he was a Visiting Scientist at the Rochester Institute of Technology and Kodak Research, Rochester, NY, where he was involved in research in optical storage channels. From 1998 to 2000, he was with the Lucent Technologies, Bell Laboratories, Murray Hill, NJ. He was involved in research coding schemes and architectures for highspeed applications, iterative decoding and low-density parity check codes, as well as development of codes and detectors implemented in Lucent (now Agere) chips. His current research interests include coding theory, information theory, communication theory, and digital communications and recording. He is cur- rently a Faculty Member at the University of Arizona, Electrical and Computer Engineering Department.

Dr. Vasic is currently a member of the Editorial Board of IEEE TRANSACTIONS ON MAGNETICS. He served as a Technical Program Chair, IEEE Communication Theory Workshop, in 2003, and as a Coorganizer of the Center for Discrete Mathematics and Theoretical Computer Science (DIMACS) Workshops on Optical/Magnetic Recording and Optical Transmission, and Theoretical Advances in Information Recording, in 2004. He was a Coorganizer of the Communication Theory Symposium within the International Conference on Communications (ICC 2006).

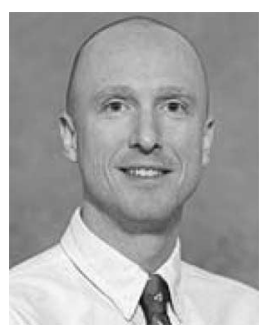

Mark A. Neifeld (S'91-M'91) received the B.S.E.E. degree from the Georgia Institute of Technology, Atlanta, in 1985 and the M.S. and Ph.D. degrees from the California Institute of Technology, Pasadena, in 1987 and 1991, respectively.

From 1985 to 1986 , he was a member of the technical staff at TRW Systems Engineering and Analysis Laboratory in Redondo Beach, CA. Following completion of his thesis work at Caltech, he accepted a one-year postdoctoral position at the NASA Jet Propulsion Laboratory in Pasadena, CA, where he studied the application of parallel image processing techniques to problems in pattern recognition. Since 1991, he was with the faculty of the Department of Electrical and Computer Engineering and the Optical Sciences Center at the University of Arizona, Tucson, AZ. He is the author or coauthor of more than 70 journal articles and more than 200 conference papers in the areas of optical communications and storage, coding and signal processing, and optical imaging and processing systems. His current research interests include information- and communication-theoretic methods in image processing, nontraditional imaging techniques that exploit the joint optimization of optical and postprocessing degrees of freedom, coding and modulation for fiber and free-space optical communications, and applications of slow and fast light. He has also been a two-term Topical Editor and a three-time Guest Editor of special issues of Applied Optics.

Prof. Neifeld is a Fellow of the Optical Society of America (OSA) and a member of the International Society for Optical Engineers (SPIE), and the American Physical Society (APS). He has served on the organizing committees of numerous conferences and symposia. 